\title{
Development and Validation of the Social Thermoregulation and Risk Avoidance Questionnaire (STRAQ-1) Supplemental Materials
}

These supplemental materials are meant to complement Vergara et al.'s STRAQ-1. It includes additional information on the methods, analysis approach, and includes some additional results.

\section{Methods}

\section{Samples}

Our samples were collected via University of Oxford (UK; $N=137,56.2 \%$ female, $\left.M_{\text {birthyear }}=1985.43 ; S D_{\text {birthyear }}=13.51\right)$, University of Belgrade (Serbia; $N=163,80.9 \%$ female, $\left.M_{\text {birthyear }}=1993.73 ; S D_{\text {birthyear }}=4.91\right)$, Singapore Management University $(N=132$, $58.3 \%$ female, $\left.M_{\text {birthyear }}=1993.80 ; S D_{\text {birthyear }}=1.53\right)$, Tsinghua University $($ China $N=174$, $62.6 \%$ female, $\left.M_{\text {birthyear }}=1993.68 ; S D_{\text {birthyear }}=6.41\right)$, University of Zürich (Switzerland; $N=$ $37,78.3 \%$ female, $\left.M_{\text {birthyear }}=1987.57 ; S D_{\text {birthyear }}=8.72\right)$, Virginia Commmonwealth University (United States; $N=148,78.3 \%$ female, $M_{\text {birthyear }}=1992.62 ; S D_{\text {birthyear }}=4.69$ ), University of Kassel (Germany; $N=103,71.8 \%$ female, $M_{\text {birthyear }}=1990.31 ; S D_{\text {birthyear }}=$ 7.83), University of California, Santa Barbara (United States; $N=106,69.8 \%$ female, $\left.M_{\text {birthyear }}=1995.82 ; S D_{\text {birthyear }}=1.71\right)$, University of Lusófona (Portugal; $N=17,41.1 \%$ female, $\left.M_{\text {birthyear }}=1984.11 ; S D_{\text {birthyear }}=11.92\right)$, University of Chile $($ Chile; $N=33,66.6 \%$ female, $M_{\text {birthyear }}=1979.33 ; S D_{\text {birthyear }}=13.16$ ), University of Southampton (United Kingdom; $N=6,50.0 \%$ female, $\left.M_{\text {birthyear }}=1992.17 ; S D_{\text {birthyear }}=1.60\right)$, Otto-Friedrichs-Universität Bamberg (Germany; $N=39,74.3 \%$ female, $M_{\text {birthyear }}=1982.10 ; S D_{\text {birthyear }}=14.66$ ), Middle East Technical University (Turkey; $N=181,65.7 \%$ female, $M_{\text {birthyear }}=1992.42 ; S D_{\text {birthyear }}=$ 5.00), University of Oslo (Norway; $N=83,71.0 \%$ female, $M_{\text {birthyear }}=1992.31 ; S D_{\text {birthyear }}=$ 6.42), and SWPS University of Social Sciences and Humanities (Poland; $N=131,90.0 \%$ female, $M_{\text {birthyear }}=1986.17 ; S D_{\text {birthyear }}=8.89$ ).

\section{Detailed Information about our Additional Questionnaires}

Questionnaires consisted of the following (information and reliabilities taken from $\mathrm{Hu}$ et al., 2019):

Perceived Stress Scale (PSS: Cohen \& Wills, 1985): The PSS is a 14-item scale ( $M$ $=2.77, S D=.55)$ measuring self-reported stress, assessing how often individuals are upset because of something that happened unexpectedly $\left(\omega_{\mathrm{t}}=.88\right.$; example item: "How often do you feel that you are unable to control the important things in your life?") and was scored from 1 ("Never") to 5 ("Very Often").

Southampton Nostalgia Scale (SNS; Sedikides, Wildschut, Arndt, \& Routledge, 2008). The SNS is a 7-item scale $(M=4.43, S D=1.33)$, where people are first explained the concept according to the dictionary, and then are ask about their experiences $\left(\omega_{\mathrm{t}}=.96\right.$; example item: "How prone are you to feeling nostalgic"). Six of the items were scored from 1 ("Not at all") to 7 ("Very Much"). The seventh item ("Specifically, how often do you bring to mind nostalgic experiences") was answered on a 7-point scale ("At least once a day", "Three to four times a week", "Approximately twice a week", "Approximately once a week", "Once or twice a month", "Once every couple of months", "Once or twice a year").

Home Attachment Scale (Harris, Brown, \& Werner, 1996). The Home Attachment Scale is a 9-item scale $(M=3.99, S D=.72)$ that assess to what degree people are attached to their home ( $\omega_{\mathrm{t}}=.77$; example item: "I feel like my home is a part of me") and is scored from 1 ("Strongly Disagree") to 5 ("Strongly Agree").

Experiences in Close Relationships (Attachment Avoidance and Anxiety; Fraley et al., 2000). The Experiences in Close Relationships Scale (overall $\omega_{\mathrm{t}}=.96$ ) is a scale to assess self-reported attachment and consists of two subscales: Attachment avoidance and attachment anxiety. The attachment anxiety subscale $(M=3.44, S D=1.12)$ consists of 18 
items $\left(\omega_{\mathrm{t}}=.93\right.$; example item: "I'm afraid that I will lose my partner's love $)$. The attachment avoidance subscale $(M=3.12, S D=1.01)$ also consists of 18 items $\left(\omega_{\mathrm{t}}=.94\right.$; example item: "I prefer not to show a partner how I feel deep down"). Both subscales were scored from 1 ("Completely Disagree") to 7 ("Completely Agree").

Daily sugary drinks consumption and diet drinks consumption (Henriksen et al., 2014). Henriksen and colleagues (2014) found in data from a large survey that married people tend to consume less sugary drinks. We borrowed the questions from their survey, which focused on fluid intake more generally. We asked participants to report their fluid consumption per day from the entire last week in numbers of cups/glasses (where 1 mug is 2 cups, 1 small plastic bottle is 4 cups, 1 large plastic bottle is 12 cups). We asked about filter coffee, instant coffee, boiled coffee, tea, herbal tea, Coca Cola/Pepsi, other fizzy drinks, Diet Cola/Diet Pepsi, other diet fizzy drinks, tap water, bottled water, juice/squash, diet juice/squash, milk, yogurt, other types of cultured milk, and other. To calculate their daily sugar drink consumption, we summed the consumption of Coca Cola/Pepsi, other fizzy drinks, and juice/squash $(M=4.59, S D=10.55)$. For the diet drinks, we summed the same, but then for their diet versions $(M=1.03, S D=3.76)$.

Nomophobia (Yildirim \& Correia, 2015). The Nomophobia scale intends to measure via 20 -items the fear of being out of cellular phone contact, which is split into a subscale of not having access to one's phone $\left(\omega_{\mathrm{t}}=.91\right.$; example item: "I would feel uncomfortable without constant access to information through my smartphone"; $M=2.86, S D=.84$ ) and not having access to one's online identity $\left(\omega_{t}=.93\right.$; example item: "If I did not have my smartphone with me, I would be worried because my family and/or friends could not reach me"; $M=2.76, S D=.78$ ). Both subscales were scored from 1 ("Strongly Disagree") to 5 ("Strongly Agree").

Self-control (Tangney, Baumeister, \& Boone, 2004). The self-control scale is a 13item scale $(M=3.14, S D=.64)$ that assesses self-reported self-control $\left(\omega_{\mathrm{t}}=.85\right.$; example item: "I am good at resisting temptation") and was scored from 1 ("Not at all") to 5 ("Very Much").

Alexithymia (Revised version of the TAS-20; Kooiman, Spinhoven, \& Trijsburg, 2002). We used a revised version of the TAS-20. The TAS-20 was originally measured on 20 items, but Kooiman et al. (2002) concluded that four of the items had insufficient loading on the subscales. We therefore only assessed the 16-item versions, with subscales Difficulty in Identifying and Describing Feelings $\left(\omega_{\mathrm{t}}=.92\right.$; example item: "I often do not know why I am angry"; $M=2.50, S D=.72)$ and Externally Oriented Thinking $\left(\omega_{\mathrm{t}}=.62\right.$; example item: "I prefer talking to people about their daily activities rather than their feelings"; $M=2.33, S D=$ .54). Both subscales were scored from 1 ("Strongly Disagree") to 5 ("Strongly Agree").

Social Network Index (Cohen, Doyle, Skoner, Rabin, \& Gwaltney, 1997). The Social Network Index assesses participation in 12 types of social relationships. These include relationships with a spouse, parents, parents-in-law, children, other close family members, close neighbours, friends, workmates, schoolmates, fellow volunteers, members of groups without religious affiliation, and religious groups. One point is assigned for each type of relationship (possible score of 12) for which respondents indicate that they speak (in person or on the phone) to persons in that relationship at least once every two weeks. The scale is scored in three different ways, for number of high contact-roles (social network diversity; $M=$ $7.40, S D=1.48$ ), number of people in the social network (a sum of all network members; $M=$ $22.64, S D=10.87)$, and the number of embedded networks people are in $(M=2.20, S D=$ 1.01). No reliability scores are available for these subscales, due to the nature of the scale. Results

Exploring STRAQ-1 's Internal Structure. We first evaluated whether our data was normally distributed through Henze-Zirkler's Multivariate Test for Normality. The data was 
not normally distributed $(H Z=1.006, p<.001)$, which should not be surprising given our multi-country sample. In such cases Principal Axis Extraction is recommended over Maximum Likelihood (Fabrigar et al., 1999), and we used both Parallel Analyses (Hayton, Allen, \& Scarpello, 2004; Raîche, Walls, Magis, Riopel, \& Blais, 2013) and Optimal Coordinates (Raîche et al., 2013) criteria to determine the number of factors to extract.

In the case of the number of factors, we used a slightly different approach than common bootstrap as we wanted to obtain a factor solution that would not be biased to a particular language or culture. Since languages and countries had different sample sizes, we tried to reduce bias (that could be caused by high sample sizes for specific countries). To do so, we ran a bootstrap-like procedure for Parallel Analyses and Optimal Coordinates, randomly selecting $50 \%$ of the sample without replacement. For each bootstrap iteration, each region and language weighted differentially as results of randomly selecting $50 \%$ of the sample without replacement. In each bootstrap iteration, each language and region present a different weight that form the original dataset. If all regions and languages would present the same solution for Parallel Analyses and Optimal Coordinates, this procedure would lead to a distribution presenting 1,000 times the same PA or OP result. However, if differences between language and regions exist, we could explore how wide is the distribution of PA an OC using this approach, selecting the solution that most frequently appeared independent of the weight of each language or region. We therefore then selected the OC and PA result that was most frequently observed across the 1,000 iterations of the bootstrap. The logic of this procedure is to keep the most likely solution given differential weights for countries and languages, therefore increasing the chances of out-of-sample replication. When discrepancies were found between Optimal Coordinates and Parallel Analysis, we examined the distributions of the results of both methods and then selected the solution that best fitted distributions for Optimal Coordinates and Parallel Analyses.

In all our factor analyses, we used oblique oblimin rotation partly as a way to investigate whether the subscales were independent or not (Costello \& Osborne, 2005). Through an iterative process running seven factor analyses we retained the least complex model while still maintaining a theoretically meaningful factor structure. In the end, we reduced the scale from 57 to 23 items, removing subloaders (items loading below 0.3 ) and crossloaders (items loading onto more than one factor) through an iterative process. This iterative process, including model fit and numbers of items removed per factor analysis, is detailed in Table 1. All factor analyses performed in these seven iterations were conducted using the bootstrap function of R package Psych (Revelle, 2018). During these iterations, Heywood cases (loadings $>1$ ) were detected. In the event Heywood cases were detected, the solution was double-checked using maximum likelihood extraction methods. In all cases results were consistent and deviations were minimal. The final solution did not present any Heywood cases anymore.

\section{Justification for Preferring Omega over Alpha}

Once we had obtained the most optimal solution through our iterative factor analyses, we evaluated each of the STRAQ-1's subscales' theoretical consistencies followed by an evaluation of reliabilities using the entire sample. In Table 2, we first report Cronbach's Alpha because it is the most well-known measure of reliability. However, given that Cronbach's Alpha is suboptimal and tends to underfit data for heterogeneous samples (Dunn, Baguley, \& Brunsden, 2014), we also report the Omega Coefficient, which does not assume variance across items and is a more robust estimate of our scales' reliabilities (Dunn et al., 2014; Revelle \& Zinbarg, 2009; Sijtsma, 2009).

Cronbach's Alpha assumes unidimensionality (meaning that all items are presumed to measure the same underlying variable), the same variance (meaning that all items are presumed to measure the underlying variable with the same empiric score magnitude), and 
covariance between items (meaning that they are presumed to equally relate to the underlying construct). These assumptions are also known as the tau-equivalent model. All or one of these assumptions are easily violated (Dunn et al., 2014), especially when the source of error can be found in translations or cultural differences. As we collected samples speaking different languages from diverse cultural regions, we expected (and found) violation of this assumption (Bartlett's K-squared $=575.36, \mathrm{df}=28, \mathrm{p}<.01$ ). A more conservative way to cope with this problem is using "Greatest Lower Bound" (GLB), which gives the lowest possible internal consistency of the scale (Sijtsma, 2009). Cronbach's alpha and GLB will over- or under correct under different situations of multidimensionality and thus over- or underfit the model, while Omega will always provide a better fit (Revelle \& Zinbarg, 2009). As such, if one has reasonable expectations to believe that the assumptions of the tau-equivalent model will be violated (such as in the case of having a heterogeneous sample) it is highly advised to rely on the Omega coefficient.

\section{External, Discriminant, and Face Validity of the STRAQ-1: Predicting Attachment from STRAQ-1 Related Habits: Analysis Procedure}

The starting model was guided by our theory and the correlations we detected. Solitary Thermoregulation was associated to attachment anxiety, Social Thermoregulation was related to attachment avoidance, while High Temperature Sensitivity, and Risk Avoidance were associated to both. We thus modeled these as predictors of attachment in our (mediation) model. Furthermore, we let attachment avoidance and anxiety co-vary, given that they are typically highly correlated (as they were in our sample; $r=.4, p<.001$ ), and let avoidance and anxiety predict stress (which in turn predicted health). In order to form our path model as robustly as we could we 1) included relevant predictor variables from our correlational studies, 2) ensured they had sufficient fit, pruning the model throughout multiple iterations. Following that, 3 ) we confirmed our model using naïve bootstrapping procedure. More concretely, we ensured that $95 \%$ CI of coefficients, obtained through bootstrapping, did not include zero (null hypothesis for coefficients in linear regression models such as path analysis). Each step of this procedure is depicted in Table 5. This pruning procedure was done following methods suggested for linear modeling (Murtaugh, 2009), which allowed us to examine whether global fit was sufficient in our path analysis.

We presumed that attachment styles should be derived from whether STRAQ-related needs are met or not. From this perspective, the STRAQ should serve as predictor for the ECR (and the ECR should not be a predictor for the STRAQ) and, if true, it increases the face validity of our assessment tool. We nevertheless also ran the model with ECR predicting STRAQ-related habits (which in turn we modelled to predict stress and health). In order to follow a comparable method as when modeling STRAQ-1 as predictor. The best model that we were able to obtain presented the following diagnostics: a significant chi square test $\left(\chi^{2}(14, \mathrm{~N}=1504)=255, \mathrm{p}<.001\right), \mathrm{CFI}=0.807, \mathrm{TLI}=0.627$, RMSEA 0.107 (C.I. $90 \%$ : $0.096-0.119)$, and SRMR $=0.065$. All fit indicators were significantly better using the ECR as mediator and STRAQ-1 as predictor $\left(\chi^{2}(5, \mathrm{~N}=1504)=214, \mathrm{p}=2.2 \mathrm{E}-16\right)$, presenting a AIC difference of 208. This further confirms our conjecture that STRAQ-1 predicts attachment styles, rather than the other way around.

\section{Cross-Sample Variability: Additional Analyses}

The STRAQ-1 could also present an opportunity to discover whether different STRAQ-1 needs weigh more heavily for attachment in some countries than in others. For example, Norwegians' attachment may be more attuned to issues surrounding to Social Thermoregulation, whereas Chinese's attachment may be more attuned to Risk Avoidance. At this time, our samples were still too small to uncover differing path analyses, while we also did not have reasonably informed hypotheses how these needs may differ in their link to attachment (and, finally, our sampling was far from representative). We therefore simply 
present radar plots in Figures 3 for countries $N>80$, presenting scores for anxiously $(M+1 S D$ on attachment anxiety), avoidantly ( $M+1 S D$ on attachment avoidance), and securely attached ( $M-1 S D$ on both attachment anxiety and avoidance) individuals in our sample. We only remark that we observe that across cultures, the links to attachment of STRAQ-1 related needs seem to differ, but future (more representative) cross-cultural research should investigate this. 


\section{Supplemental Material References}

Cohen, S., \& Wills, T. A. (1985). Stress, social support, and the buffering hypothesis. Psychological Bulletin, 98, 310-357. https://doi.org/10.1037/0033-2909.98.2.310

Cohen, S., Doyle, W. J., Skoner, D. P., Rabin, B. S., \& Gwaltney, J. M. (1997). Social ties and susceptibility to the common cold. Jama, 277, 1940-1944. doi.org/10.1001/jama.1997.03540480040036

Costello, A. B. A. B., \& Osborne, J. W. J. W. (2005). Best practices in exploratory factor analysis: Four recommendations for getting the most from your analysis. Practical Assessment, Research \& Evaluation, 10, 1-9. Retrieved from http://citeseerx.ist.psu.edu/viewdoc/download?doi=10.1.1.110.9154\&amp;rep=rep1\&a mp;type $=$ pdf

Dunn, T. J., Baguley, T., \& Brunsden, V. (2014). From alpha to omega: A practical solution to the pervasive problem of internal consistency estimation. British Journal of Psychology, 105, 399-412. https://doi.org/10.1111/bjop.12046

Fabrigar, L. R., Wegener, D. T., MacCallum, R. C., \& Strahan, E. J. (1999). Evaluating the use of exploratory factor analysis in psychological research. Psychological Methods, 4, 272-299. https://doi.org/10.1037/1082-989X.4.3.272

Fraley, R. C., Waller, N. G., \& Brennan, K. A. (2000). An item response theory analysis of self-report measures of adult attachment. Journal of Personality and Social Psychology, 78, 350-365. https://doi.org/10.1037/0022-3514.78.2.350

Harris, P. B., Brown, B. B., \& Werner, C. M. (1996). Privacy regulation and place attachment: Predicting attachments to a student family housing facility. Journal of Environmental Psychology, 16, 287-301. https://doi.org/10.1006/jevp.1996.0025

Hayton, J. C., Allen, D. G., \& Scarpello, V. (2004). Factor retention decisions in Exploratory Factor Analysis: A tutorial on Parallel Analysis. Organizational Research Methods, 7, 191-205. https://doi.org/10.1177/1094428104263675

Henriksen, R. E., Torsheim, T., \& Thuen, F. (2014). Loneliness, social integration and consumption of sugar-containing beverages: Testing the Social Baseline Theory. PLoS ONE, 9. https://doi.org/10.1371/journal.pone.0104421

Hu, C. P., Yin, J. X., Lindenberg, S., Dalğar, İ., Weissgerber, S. C., Vergara, R. C., ... \& IJzerman, H. (2019). Data from the Human Penguin Project, a cross-national dataset testing social thermoregulation principles. Scientific Data, 6, 32. https://doi.org/10.1038/s41597-019-0029-2

Kooiman, C. G., Spinhoven, P., \& Trijsburg, R. W. (2002). The assessment of alexithymia: A critical review of the literature and a psychometric study of the Toronto Alexithymia Scale-20. Journal of Psychosomatic Research, 53, 1083-1090. https://doi.org/10.1016/S0022-re3999(02)00348-3

Murtaugh, P. A. (2009). Performance of several variable-selection methods applied to real ecological data. Ecology Letters, 12, 1061-1068. https://doi.org/10.1111/j.14610248.2009.01361.x

Revelle, W. (2018). Psych: Procedures for personality and psychological research, version 1.8.3. Retrieved from https://cran.r-project.org/package $=$ psych

Raîche, G., Walls, T. A., Magis, D., Riopel, M., \& Blais, J.-G. (2013). Non-graphical solutions for Cattell's scree test. Methodology, 9, 23-29. https://doi.org/10.1027/16142241/a000051

Revelle, W., \& Zinbarg, R. E. (2009). Coefficients Alpha, Beta, Omega, and the glb: Comments on Sijtsma. Psychometrika, 74, 145-154. https://doi.org/10.1007/s11336$\underline{008-9102-z}$ 
Sedikides, C., Wildschut, T., Arndt, J., \& Routledge, C. (2008). Nostalgia: Past, present, and future. Current Directions in Psychological Science, 17, 304-307. https://doi.org/10.1111/j.1467-8721.2008.00595.x

Sijtsma, K. (2009). On the use, the misuse, and the very limited usefulness of Cronbach's alpha. Psychometrika, 74, 107-120. https://doi.org/10.1007/s11336-008-9101-0

Tangney, J. P., Baumeister, R. F., \& Boone, A. L. (2004). High self-control predicts good adjustment, less pathology, better grades, and interpersonal success. Journal of Personality, 72, 271-324. https://doi.org/10.1111/j.0022-3506.2004.00263.x

Yildirim, C., \& Correia, A. P. (2015). Exploring the dimensions of nomophobia: Development and validation of a self-reported questionnaire. Computers in Human Behavior, 49, 130-137. https://doi.org/10.1016/j.chb.2015.02.059 
Figure 3a. Radar Plots for each STRAQ-1 subscale by language and attachment style.

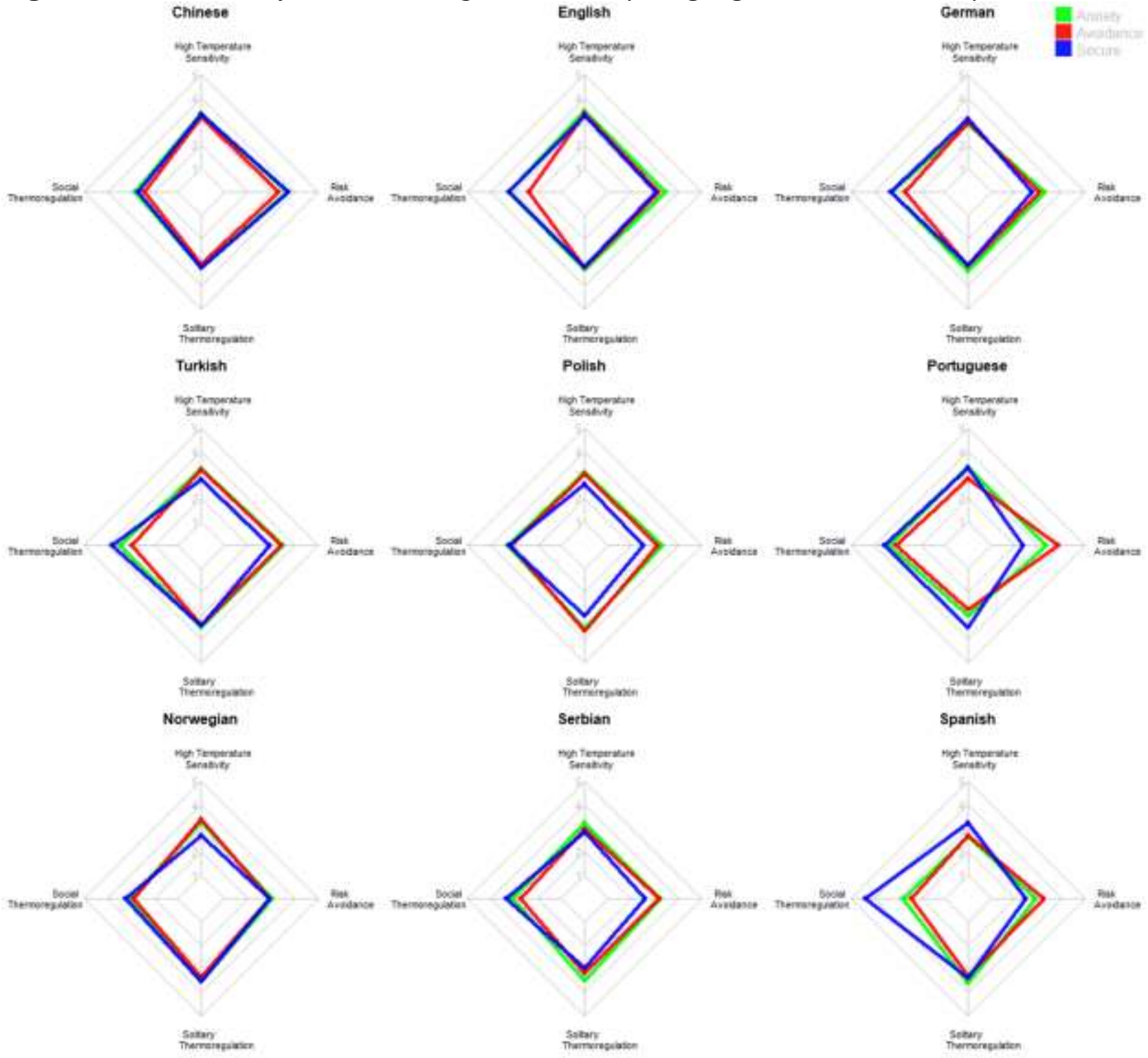


Appendix

A.1

\begin{tabular}{|c|c|}
\hline Items & \\
\hline STRAQ1 & I am not sensitive to coldness \\
\hline STRAQ2 & I am sensitive to heat \\
\hline STRAQ3 & I find cool days pleasant \\
\hline STRAQ4 & I find warm days pleasant \\
\hline STRAQ5 & I find hot days pleasant \\
\hline STRAQ6 & When it is cold, I more quickly turn up the heater than others \\
\hline STRAQ7 & When it is warm, I more quickly want to go to the pool than others \\
\hline STRAQ8 & When it is hot, I wear less clothing than others \\
\hline STRAQ9 & When it is cold, I wear more clothing than others \\
\hline STRAQ10 & I don't like when it's too hot \\
\hline STRAQ11 & When I feel warm I do not want to do anything \\
\hline STRAQ12 & I never feel my hands cold \\
\hline STRAQ13 & I prefer to work under cold environments rather than hot \\
\hline STRAQ14 & I can't focus when it is too hot \\
\hline STRAQ15 & I can't focus when it is too cold \\
\hline STRAQ16 & I prefer to relax in a cold place \\
\hline STRAQ17 & I don't like to engage in sports on cold days \\
\hline STRAQ18 & I like to take a nap on hot days \\
\hline STRAQ19 & I usually have more physical contact with others than most people \\
\hline STRAQ20 & When people are close to me, I like to be really close to them \\
\hline STRAQ21 & I like to spend time alone \\
\hline STRAQ22 & I do not like crowd places \\
\hline STRAQ23 & I like to meet new friends \\
\hline STRAQ24 & I love to cuddle \\
\hline STRAQ25 & I like to hug my friends \\
\hline STRAQ26 & When I am with my partner, I feel energized \\
\hline STRAQ27 & When I am with my parents, I feel energized \\
\hline STRAQ28 & When I am with other people in general, I feel energized \\
\hline STRAQ29 & I feel energized after sharing my emotions with my partner \\
\hline STRAQ30 & I feel energized after sharing my emotions with my parents \\
\hline STRAQ31 & I feel tired if I think about spending time with my partner \\
\hline STRAQ32 & I feel tired if I think about spending time with my parents \\
\hline STRAQ33 & When my partner is away I feel more vigorous \\
\hline STRAQ34 & I always carry extra clothing \\
\hline STRAQ35 & I always check the weather forecast \\
\hline STRAQ36 & I am always attentive of what's happening around me \\
\hline STRAQ37 & I plan my travels without leaving anything to luck \\
\hline STRAQ38 & I love to arrive to unknown places alone \\
\hline STRAQ39 & I never consider backup plans in case something may happen \\
\hline STRAQ40 & I try to maintain myself in familiar places \\
\hline STRAQ41 & I don't trust people I have not met before \\
\hline STRAQ42 & I don't avoid asking instructions from unfamiliar people \\
\hline
\end{tabular}




\begin{tabular}{ll}
\hline STRAQ43 & I try to be accompanied by people that I know at all times \\
STRAQ44 & When I feel cold I seek someone to cuddle with \\
STRAQ45 & When I feel cold I don't turn on the heater \\
STRAQ46 & I love to spend time with my family or friends when it's cold out \\
STRAQ47 & During cold days I just grab a blanket \\
STRAQ48 & I like to warm up my hands or feet by touching someone who I am close to \\
STRAQ49 & I prefer to warm up with someone rather than with something \\
STRAQ50 & I love to share a hot beverage with a friend when I am feeling down \\
STRAQ51 & When I am upset I don't seek for someone close to be with \\
STRAQ52 & When I am troubled I like to take a long warm shower to clear up my thoughts \\
STRAQ53 & When I am distressed I really need a "warm shoulder" \\
STRAQ54 & A warm beverage always helps me relax when I am down \\
STRAQ55 & If am feeling distressed I seek a warm place to calm down \\
STRAQ56 & If I am feeling down I don't like to stay in bed \\
STRAQ57 & When I am feeling sad I like to hug a pillow or a teddy bear \\
\hline
\end{tabular}




\section{Appendix B.1 STRAQ-1 Info Sheet}

This sheet was written by Justin Mah, Rodrigo Vergara, Rick Klein, and Hans IJzerman

\section{Researcher General Information}

The STRAQ-1 was designed to understand the motivations underlying interpersonal bonding (i.e., attachment). A secondary goal was to investigate whether adding the STRAQ-1's items could add explained variance in self-reported health and stress over attachment alone. The current model was formed through iterative analyses (cross-validation and naive

bootstrapping) of datasets that have been collected from (12) different countries. The scale is currently available in Portuguese, Polish, Turkish, Mandarin, German, English, Norwegian, Serbian and Spanish. The STRAQ-1 has four subscales to predict attachment: High Temperature Sensitivity, Social Thermoregulation, Solitary Thermoregulation, and Risk Avoidance. Furthermore, we also related the STRAQ-1 subscales to other factors, such as social network size, social embeddedness, attachment style, stress and self-reported health. The following project page has more detailed explanation of the data collection process of the current STRAQ-1 (https://osf.io/2rm5b/). 


\begin{tabular}{|c|c|c|c|c|}
\hline & $\begin{array}{l}\text { HIGH } \\
\text { TEMPERATU } \\
\text { RE } \\
\text { SENSITIVITY }\end{array}$ & $\begin{array}{l}\text { SOCIAL } \\
\text { THERMOREGULAT } \\
\text { ION }\end{array}$ & $\begin{array}{l}\text { SOLITARY } \\
\text { THERMOREGULAT } \\
\text { ION }\end{array}$ & $\begin{array}{l}\text { RISK } \\
\text { AVOIDANCE }\end{array}$ \\
\hline GOAL & $\begin{array}{l}\text { This subscale } \\
\text { measures } \\
\text { individual } \\
\text { differences in } \\
\text { sensitivity to } \\
\text { high ambient } \\
\text { temperature. } \\
\text { Measures } \\
\text { comfort and } \\
\text { discomfort in } \\
\text { relation to } \\
\text { temperature. }\end{array}$ & $\begin{array}{l}\text { This subscale measures } \\
\text { individual differences } \\
\text { in the desire to warm up } \\
\text { with someone on a } \\
\text { regular basis when cold } \\
\text { or when feeling } \\
\text { distressed. }\end{array}$ & $\begin{array}{l}\text { This subscale measures } \\
\text { individual differences } \\
\text { in the desire to warm up } \\
\text { without the aid of } \\
\text { others when feeling } \\
\text { cold or distressed. }\end{array}$ & $\begin{array}{l}\text { This subscale } \\
\text { measures } \\
\text { individual } \\
\text { differences in } \\
\text { the tendency to } \\
\text { avoid (social) } \\
\text { exploration. }\end{array}$ \\
\hline $\begin{array}{l}\text { HOW TO } \\
\text { INTERPRET }\end{array}$ & $\begin{array}{l}\text { Higher scores } \\
\text { indicate greater } \\
\text { discomfort when } \\
\text { the environment } \\
\text { is too hot (and } \\
\text { greater } \\
\text { preference for } \\
\text { cold } \\
\text { environments.) }\end{array}$ & $\begin{array}{l}\text { Higher scores indicate } \\
\text { greater desire to } \\
\text { socially thermoregulate } \\
\text { when cold or distressed. }\end{array}$ & $\begin{array}{l}\text { Higher scores indicate } \\
\text { greater desire to } \\
\text { thermoregulate } \\
\text { solitarily when cold or } \\
\text { distressed. }\end{array}$ & $\begin{array}{l}\text { Higher scores } \\
\text { indicate a lower } \\
\text { likelihood to } \\
\text { explore (social) } \\
\text { situations. }\end{array}$ \\
\hline $\begin{array}{l}\text { CLASSIFICA } \\
\text { TION } \\
\text { LEVELS }^{1}\end{array}$ & $\begin{array}{l}\text { Low 1.00-2.70 } \\
\text { Medium 2.7- } \\
3.57 \\
\text { High 3.57-5.00 }\end{array}$ & $\begin{array}{l}\text { Low 1.00-2.60 } \\
\text { Medium 2.60-3.60 } \\
\text { High 3.60-5.00 }\end{array}$ & $\begin{array}{l}\text { Low 1.00-2.87 } \\
\text { Medium 2.87-3.75 } \\
\text { High 3.75-5.00 }\end{array}$ & $\begin{array}{l}\text { Low 1.00-2.67 } \\
\text { Medium 2.67- } \\
3.67 \\
\text { High 3.67-5.00 }\end{array}$ \\
\hline
\end{tabular}

\footnotetext{
${ }^{1}$ The classification levels are designated with low being the bottom quartile of scores, medium being the 2nd and 3rd quartiles in range, and high being the top quartile of scores
} 


\section{Researcher Information: STRAQ-1 (The Questionnaire)}

Instructions to provide to participants: Below is a list of statements. Please read each statement carefully and rate how frequently you feel or act in the manner described. If you strongly disagree with the statement, please select 1; if you disagree, please select 2; if you don't feel like you agree or disagree, please select 3; if you agree select 4; finally, if you strongly agree select 5 . There are no right or wrong answers, we are solely interested in how you feel. If you are not currently in a dating or marital relationship with someone, answer the questions pertaining to a partner with respect to a former partner or a relationship that you would like to have with someone.

\section{STRAQ-1 Items (" notes reverse scored)}

\begin{tabular}{|c|c|}
\hline \multirow{7}{*}{$\begin{array}{l}\text { High Temperature } \\
\text { Sensitivity }\end{array}$} & I am sensitive to heat \\
\hline & I find warm days pleasant* \\
\hline & I find hot days pleasant* \\
\hline & I don't like when it's too hot \\
\hline & When I feel warm I do not want to do anything \\
\hline & I can't focus when it is too hot \\
\hline & I prefer to relax in a cold place \\
\hline \multirow{5}{*}{$\begin{array}{l}\text { Social } \\
\text { Thermoregulation }\end{array}$} & I usually have more physical contact with others than most people \\
\hline & When people are close to me, I like to be really close to them \\
\hline & When I feel cold I seek someone to cuddle with \\
\hline & I like to warm up my hands or feet by touching someone who I am close 1 \\
\hline & I prefer to warm up with someone rather than with something \\
\hline Solitary & I am not sensitive to coldness* \\
\hline \multirow[t]{7}{*}{ Thermoregulation } & When it is cold, I more quickly turn up the heater than others \\
\hline & When it is cold, I wear more clothing than others \\
\hline & I can't focus when it is too cold. \\
\hline & When I feel cold I don't turn on the heater* \\
\hline & When I am troubled I like to take a long warm shower to clear up my tho \\
\hline & A warm beverage always helps me relax when I am down \\
\hline & If I am feeling distressed I seek a warm place to calm down \\
\hline \multirow[t]{3}{*}{ Risk Avoidance } & I try to maintain myself in familiar places \\
\hline & I don't trust people I have not met before \\
\hline & I try to be accompanied by people that I know at all times \\
\hline
\end{tabular}

\section{STRAQ-1 Items (Short Version)}

\begin{tabular}{l|l}
\hline $\begin{array}{l}\text { High Temperature } \\
\text { Sensitivity }\end{array}$ & $\begin{array}{l}\text { I find hot days pleasant* } \\
\text { I don't like when it's too hot }\end{array}$ \\
$\begin{array}{l}\text { Social } \\
\text { Thermoregulation }\end{array}$ & I prefer to warm up with someone rather than with something \\
Solitary & I like to warm up my hands or feet by touching someone who I am close 1 \\
Thermoregulation & When it is cold, I more quickly turn up the heater than others \\
Risk Avoidance & I try to maintain myself in familiar places \\
& I try to be accompanied by people that I know at all times
\end{tabular}




\section{STRAQ-1 Participant Feedback}

In some cases (e.g., in online research) it is desirable to provide participants with feedback. Below you will find brief feedback paragraphs, based on the analyses and norm scores from our 12-country dataset.

\section{High Temperature Sensitivity:}

This scale measures how sensitive a person is to higher ambient temperatures. It was designed to measure how comfortable an individual is with higher ambient (subjective) temperatures. Scoring higher on this scale typically means you have a greater discomfort with warmer environments (and a greater preference to the cold).

What your scores mean: Based on an average of how people in 12 different countries have scored, the scale has been divided into low, medium, and high temperature sensitive individuals. The lowest quarter of scores are considered "less sensitive" to hotter temperatures, while the highest quarter of scores are considered "highly sensitive" to hotter temperatures. The rest of the scores in the middle are considered "moderately sensitive" to hotter temperatures.

What you scored: You scored ..., which is between 1.00-2.70. This is the lower quartile of scores. People with these scores are considered "less sensitive" to hotter temperatures. People who score lower on the subscale of High Temperature Sensitivity tend to have less attachment anxiety. They also tend to have lower self-reported stress and better self-reported health.

What you scored: You scored ... which is between 2.70-3.57. This is in the middle of all scores. People with these scores are considered to be "moderately sensitive" to hotter temperatures. More generally speaking, people who score higher on the subscale of High Temperature Sensitivity tend to have less attachment anxiety (but people who score moderate on this scale thus tend to have moderate attachment anxiety). They also tend to have higher self-reported stress and worse self-reported health (and people who score moderate on this scale thus tend to have moderate self-reported stress and moderate levels of self-reported health).

What you scored: You scored ... which is between 3.57-5.00. This is the higher quartile of scores. People with these scores are considered "highly sensitive" to hotter temperatures. People who score higher on the subscale of High Temperature Sensitivity tend have more attachment anxiety. They also tend to have higher self-reported stress and worse self-reported health.

\section{Social Thermoregulation:}

This scale measures a person's desire to seek the aid of someone else to warm up regularly when they are feeling either cold or distressed. It was designed to measure how much people desire to rely on social strategies to regulate their own temperature. Scoring higher on this scale typically means you have a higher desire to seek out others when cold or distressed. What your scores mean: Based on an average of how people in 12 different countries have scored, the scale has been divided into low, medium, and high temperature sensitive individuals. The lowest quarter of scores are considered "low desire for Social Thermoregulation" when cold or distressed, while the highest quarter of scores are considered "high desire for Social Thermoregulation" when cold or distressed. The rest of the scores in the middle are considered "medium desire for Social Thermoregulation" when cold or distressed. 
What you scored: You scored ... which is between 1.00-2.60. This is the lower quartile of people. People with these scores are considered "low desire for Social Thermoregulation" when it comes to being cold or distressed. People who score lower on the subscale of Social Thermoregulation tend have more attachment avoidance. They also tend to have worse selfreported health.

What you scored: You scored ... which is between 2.60-3.60. This is in the middle of all scores. People within these scores are considered around "medium desire for Social Thermoregulation" in response to cold or distress. More generally speaking, people who score higher on the subscale of Social Thermoregulation tend to have less attachment avoidance (thus, people who score moderate on this scale tend have moderate attachment avoidance). They also tend to have better self-reported health (thus, people who score moderate on this scale tend to have moderate levels of self-reported health).

What you scored: You scored ... which is between 3.60-5.00. This is the higher quartile of people. People with these scores are considered "high desire for social thermoregulation" in response to cold or distress. More generally speaking, people who score higher on the subscale of Social Thermoregulation tend have less attachment avoidance. They also tend to have better self-reported health.

\section{Solitary Thermoregulation:}

This scale measures an individual's desire to warm up without the help of others when they are feeling cold or distressed. It was designed to measure a person's desire to regulate their own temperature independently of others. Scoring higher on this scale typically means you have a higher desire to regulate your temperature independent of others.

What your scores means: Based on an average of how people in 12 different countries have scored, the scale has been divided into low, medium, and high Solitary Thermoregulation individuals. The lowest quarter of scores are considered "low desire for Solitary Thermoregulation" in response to cold or distress, while the highest quarter of scores are considered "high desire for Solitary Thermoregulation" in response to cold or distress. The rest of the scores in the middle are considered "medium desire for Solitary Thermoregulation" to cold or distress.

What you scored: You scored ... which is between 1.00-2.87. This is the lower quartile of score. People with these scores are considered to have "low desire for Solitary Thermoregulation" in response to cold or distress. People who score lower on the subscale of Solitary Thermoregulation tend have less attachment anxiety and tend to report feeling less stress.

What you scored: You scored ... which is between 2.87-3.75. This is in the middle of all scores. People with these scores are considered to have "medium desire for Solitary Thermoregulation" in response to cold or distress. More generally speaking, people who score higher on the subscale of Solitary Thermoregulation tend to have less attachment anxiety and tend to report feeling more stress (thus, people who score moderate on this scale tend to have moderate attachment anxiety and report moderate levels of stress as well).

What you scored: You scored ... which is between 3.75-5.00. This is the higher quartile of scores. People with these scores are considered to have "high desire for Solitary Thermoregulation" in response to cold or distress. People who score higher on the subscale of 
Solitary Thermoregulation tend to have more attachment anxiety and tend to report feeling more stress.

\section{Risk Avoidance:}

This scale measures an individual's tendency for social exploration. Higher scores in Risk Avoidance are typically linked to a lower likelihood of that person exploring social situations. What the scores mean: Based on an average of how people in 12 different countries have scored, the scale has been divided into low, medium, and high Risk Avoidance individuals. The lowest quarter of scores are considered "low Risk Avoidance" to social exploration, while the highest quarter of scores are considered "high Risk Avoidance" to social exploration. The rest of the scores in the middle are considered "medium Risk Avoidance" to social exploration as they are between the lowest and highest quartile of people.

What you scored: You scored ... which is between $1.00-2.67$. This is the lower quartile of all scores. People with these scores are considered to have "low Risk Avoidance" to social exploration. More generally, people who score lower on the Risk Avoidance subscale tend have more attachment anxiety. They tend to have lower self-reported stress and better selfreported health.

What you scored: You scored ... which is between 2.67- 3.67. This is in the middle of all scores. People with these scores are considered to have "medium Risk Avoidance" to social exploration. More generally, people who score higher on the Risk Avoidance subscale tend have more attachment anxiety (and people who score moderate thus tend to have moderate attachment anxiety). They tend to have higher self-reported stress, and worse self-reported health (and people who score moderate on this scale thus tend to have moderate levels of selfreported health and stress).

What you scored: You scored ... which is between 3.67-5.00. This is the higher quartile of all scores. People with these scores are considered to have "high Risk Avoidance" to social exploration. More generally, people who score higher on the Risk Avoidance subscale tend to have more attachment anxiety. They tend to have higher self-reported stress, and worse selfreported health. 